\title{
Principais classes farmacológicas relacionadas à farmacodermia
}

\author{
Main pharmacological classes related to pharmacodermia \\ Principales clases farmacológicas relacionadas con la farmacodermia
}

Recebido: 07/01/2022 | Revisado: 13/01/2022 | Aceito: 16/01/2022 | Publicado: 18/01/2022

Maria Eduarda Fernandes de Melo

ORCID: https://orcid.org/0000-0001-7768-3557

Faculdade Unibras de Goiás, Brasil

E-mail: mariaeduardamfm@gmail.com

Cínthia Alves Porfiro

ORCID: https://orcid.org/0000-0002-4398-0323

Faculdade Unibras de Goiás, Brasil

E-mail: cinthiaporfiro@hotmail.com

Luciana Arantes Dantas

ORCID: https://orcid.org/0000-0001-8138-4824 Faculdade Unibras de Goiás, Brasil

E-mail: luciana.dantas@unibras.digital

Manoel Aguiar Neto Filho

ORCID: https://orcid.org/0000-0002-3545-8746

Faculdade Unibras de Goiás, Brasil

E-mail: manuel-aguiar@ hotmail.com

Celiana Maria Ferrarini Triches

ORCID: https://orcid.org/0000-0002-8031-3837

Faculdade Unibras de Goiás, Brasil

E-mail: celianaferrarini@hotmail.com

Jacqueline da Silva Guimarães dos Santos

ORCID: https://orcid.org/0000-0002-0993-1114

Faculdade Unibras de Goiás, Brasil

E-mail: guimaraes.js@alumni.usp.br

\begin{abstract}
Resumo
Farmacodermia é uma reação dermatológica adversa a algum fármaco, ou seja, qualquer efeito colateral relacionado à pele e/ou a seus anexos como, mucosas, cabelos e unhas, na sua estrutura ou função. Medicamentos contendo fármacos que podem causar farmacodermias são desconhecidos pela população. As farmacodermias estão entre as reações adversas a medicamentos mais comuns, assumindo múltiplos aspectos clínicos, desde lesões solitárias até quadros generalizados. Nesse sentido trazer informações úteis para os leitores sobre a ocorrência de casos de farmacodermia nos últimos anos e fazer um levantamento das principais classes farmacológicas responsáveis é de suma importância pois as farmacodermias estão cada vez mais frequentes, causando grande desconforto e dor aos pacientes. O trabalho foi elaborado através de uma revisão sistemática de literatura sobre a farmacodermia. A pesquisa foi realizada utilizando as palavras-chave farmacodermia, nos portais Google Acadêmico e Portal Regional da BVS (Biblioteca Virtual em Saúde) que utilizam as bases de dados do Scientific Electronic Library Online (SCIELO), Literatura Latino-Americana e do Caribe em Ciências da Saúde (LILACS), Medical Literature Analysis and Retrieval System Online (MEDLINE) entre outras, resultando em 153 trabalhos científicos. Verificamos que as classes farmacológicas de maior incidência em casos de farmacodermias foram os anti-inflamatórios, quimioterápicos, anticonvulsivantes, ansiolíticos, anti-hipertensivos e antibióticos, contudo toda medicação pode causar efeitos adversos do tipo farmacodermias. Essas classes estão entre as mais prescritas em todo mundo, portanto, o contato da equipe multiprofissional e a farmácia clínica são essenciais para a rápida identificação de possíveis farmacodermias.
\end{abstract}

Palavras-chave: Farmacodermias; Reações adversas dermatológicas; Efeitos colaterais dermatológicos.

\begin{abstract}
Pharmacoderma is an adverse dermatological reaction to a drug, wich is, any side effect related to the skin and/or its adnexa, such as mucous membranes, hair and nails, in its structure or function. Medicines containing drugs that can cause pharmacoderma are unknown to the population. Pharmacoderma are among the most common adverse drug reactions, assuming multiple clinical aspects, from solitary lesions to generalized conditions. In this sense, bringing useful information to readers about the occurrence of pharmacoderma cases in recent years and making a survey of the main responsible pharmacological classes is of paramount importance because pharmacoderma is increasingly frequent, causing great discomfort and pain to patients. The work was carried out through a systematic review of the literature on pharmacoderma. The search was carried out using the keywords pharmacodermia, in the Google Academic and Regional Portal of the VHL (Virtual Health Library) using the Scientific Electronic Library Online
\end{abstract}


(SCIELO) databases, Latin American and Caribbean Literature in Science of Health (LILACS), Medical Literature Analysis and Retrieval System Online (MEDLINE), among others, resulting in 153 scientific papers. We found that the pharmacological classes with the highest incidence in cases of pharmacoderma were anti-inflammatory, chemotherapeutic, anticonvulsant, anxiolytic, antihypertensive and antibiotic, however all medication can cause adverse effects such as pharmacoderma. These classes are among the most prescribed in the world, therefore, the contact of the multidisciplinary team and the clinical pharmacy are essential for the rapid identification of possible pharmacoderma.

Keywords: Pharmacodermias; Dermatological adverse reactions; Dermatological side effects.

\section{Resumen}

La farmacodermia es una reacción dermatológica adversa a un fármaco, es decir, cualquier efecto secundario relacionado con la piel y / o sus anexos, como mucosas, cabello y uñas, en su estructura o función. Los medicamentos que contienen fármacos que pueden causar farmacodermia son desconocidos para la población. La farmacodermia se encuentra entre las reacciones adversas a medicamentos más comunes, asumiendo múltiples aspectos clínicos, desde lesiones solitarias hasta condiciones generalizadas. En este sentido, acercar información útil a los lectores sobre la ocurrencia de casos de farmacodermia en los últimos años y realizar un relevamiento de las principales clases farmacológicas responsables es de suma importancia porque la farmacodermia es cada vez más frecuente, provocando un gran malestar y dolor a los pacientes. El trabajo se llevó a cabo mediante una revisión sistemática de la literatura sobre farmacodermia. La búsqueda se realizó mediante las palabras clave farmacodermia, en el Portal Académico y Regional Google de la BVS (Biblioteca Virtual en Salud) utilizando las bases de datos de la Biblioteca Electrónica Científica en Línea (SCIELO), Literatura Latinoamericana y del Caribe en Ciencias de la Salud (LILACS), Sistema de Análisis y Recuperación de Literatura en Línea (MEDLINE), entre otros, dando como resultado 153 artículos científicos. Encontramos que las clases farmacológicas con mayor incidencia en casos de farmacodermia fueron antiinflamatorios, quimioterápicos, anticonvulsivos, ansiolíticos, antihipertensivos y antibióticos, sin embargo toda medicación puede ocasionar efectos adversos como la farmacodermia. Estas clases se encuentran entre las más prescritas en el mundo, por lo que el contacto del equipo multidisciplinar y la farmacia clínica son fundamentales para la rápida identificación de una posible farmacodermia.

Palabras clave: Farmacoderma; Reacciones adversas dermatológicas; Efectos secundarios dermatológicos.

\section{Introdução}

Farmacodermia é uma reação dermatológica adversa a algum fármaco, ou seja, qualquer efeito colateral relacionado à pele e/ou a seus anexos como, mucosas, cabelos e unhas, na sua estrutura ou função (Sociedade Brasileira de Dermatologia, 2017).

Cerca de 5 a $10 \%$ de todos os pacientes que utilizam medicamentos irão desenvolver alguma forma de Farmacodermia. No Brasil isso pode estar ocorrendo pela facilidade de aquisição ou mesmo pela imprudência na prescrição de medicamentos. As farmacodermias podem se manifestar como sintomas mais simples na pele ou atingir estágio mais avançado podendo levar o indivíduo a morte, por exemplo, no caso da Síndrome de Stevens-Johnson e a necrose epidérmica tóxica (Tavares et al., 2016).

Medicamentos contendo fármacos que podem causar farmacodermias são desconhecidos pela população. As farmacodermias estão entre as reações adversas a medicamentos mais comuns, assumindo múltiplos aspectos clínicos, desde lesões solitárias até quadros generalizados (Quaresma et al., 2016).

Farmacodermia são reações adversas, a medicamentos comuns que na prática médica, estando presente em $2 \%$ de todos os métodos de tratamento. Existem muitos tipos de reações medicamentosas, a maioria das quais produz uma erupção cutânea moderada. As síndromes cutâneas graves que podem ser causadas por medicamentos são a síndrome de StevensJohnson (SJS) e a necrólise epidérmica tóxica (NET) e ainda a síndrome de Lyell, que envolve a pele e as membranas mucosas. Atualmente, todas essas síndromes são consideradas espectros de uma mesma doença, distinguindo-se pela expansão da área cutânea afetada (Andrade et al., 2019).

Nesse sentido trazer informações úteis para os leitores sobre a ocorrência de casos de farmacodermia nos últimos anos e fazer um levantamento das principais classes farmacológicas responsáveis e de suma importância, pois as farmacodermias estão cada vez mais frequentes, causando grande desconforto e dor aos pacientes. 
A Organização Mundial de Saúde (OMS, 2015), define reação adversa a medicamentos (RAM) como:

"qualquer efeito prejudicial ou indesejável, não intencional, que aparece após a administração de um medicamento em doses normalmente utilizadas no homem para a profilaxia, o diagnóstico e o tratamento de uma enfermidade".

Estima-se que as reações adversas medicamentosas ocupam entre o quarto e sexto lugar como causa de morte em alguns países, a pele, como órgão imunológico, participa de maneira ativa na fisiopatologia dessas reações (Innocencio et al, 2017).

Farmacodermia é uma reação dermatológica adversa a determinados fármacos, ou seja, qualquer efeito colateral relacionado à pele e/ou a seus anexos como, mucosas, cabelos e unhas, na sua estrutura ou função. Essa reação pode ocorrer por dose excessiva de fármaco, dose usual do fármaco devido reação individual (idiossincrasia), reação alérgica ou interação medicamentosa. Quando ocorre por reação alérgica é resultado de mecanismos imunológicos denominados reações de hipersensibilidade a fármacos, não dependente de dose e sim da susceptibilidade do indivíduo, podendo apresentar sinais clínicos restritos à pele ou de forma sistêmica (Figueiredo et al., 2017; Sociedade Brasileira de Dermatologia, 2017).

A farmacodermia devido reações de hipersensibilidade está entre os tipos mais severos de reação a medicamentos no qual o efeito cutâneo se dá por reação alérgica, com presença de anticorpos IgE específicos a um fármaco, por exemplo contra antígenos $\beta$-lactâmicos. Estas reações não dependem da dose e surgem em algumas pessoas que desenvolvem susceptibilidade a determinados medicamentos, ou grupo deles, mesmo que tenham tomado a medicação anteriormente e não tenham desenvolvido efeito adverso inicial. Contudo algumas farmacodermias não dependem do sistema imunológico, podem acontecer em qualquer pessoa e em qualquer idade devido a propriedades farmacológicas ou interações medicamentosas (Pires et al., 2017).

Santos et al. (2015) em período inferior a um ano identificaram uma variedade de farmacodermias em internados em clínica dermatológica: DRESS (do inglês, Drug Reaction with Eosinophilia and Systemic Symptoms Syndrome, tradução literal, reação a fármaco com eosinofilia e Síndrome sintomática sistêmica), fotossensibilidade, necrólise epidérmica tóxica, dermatose bolhosa, reação de hipersensibilidade, anafilaxia, psoríase eritrodérmica, anemia hemolítica com reação de hipersensibilidade, dermografia, síndrome de Stevens-Johnson.

Diferentes classes farmacológicas estão relacionadas a possíveis farmacodermias em diferentes perfis de pacientes, de recém-nascidos a idosos, como por exemplo: anti-inflamatórios não esteroides (AINES), antibióticos, anticonvulsivantes, antidiabéticos, anti-hipertensivos, antipsicóticos, diuréticos, imunossupressores, entre outros. Portanto, o contato do paciente com equipe multiprofissional e a farmácia clínica são essenciais para a rápida identificação de possíveis farmacodermias (Santos et al., 2015; Andrade et al., 2019).

As reações adversas a medicamentos são um problema importante na prática médica porque causam hospitalização, hospitalização prolongada morbidade ou mortalidade. Além disso, modificam negativamente a qualidade de vida do paciente, contribuem para a perda de confiança do paciente na equipe de saúde, aumentam despesas, também pode atrasar o tratamento, pois pode simular doenças (Figueiredo et al., 2017).

Algumas características indicam maior risco de gravidade nas farmacodermias, como presença de eritema confluente, edema facial, lesões cutâneas dolorosas, púrpura palpável, necrose, bolhas ou destacamento epidérmico, erosões mucosas, febre alta, linfadenopatia, artralgias ou artrite, eosinofilia, linfocitose atípica e elevação de enzimas hepáticas, sugerem evolução desfavorável. A ocorrência destas alterações nem sempre é precoce, fazendo-se necessária a identificação de outros achados que permitam a rápida detecção de sinais de alerta. Por mais que alguns achados clínicos possam ser importantes preditores para o prognóstico das farmacodermias, nenhum algoritmo provou ser sensível e específico e há casos em que o desfecho é pouco previsível (Rossi, 2017). 
Os sintomas das farmacodermias geralmente aparecem cerca de dois dias após o contato com o medicamento, aparecem manchas avermelhadas na pele, acompanhadas de coceira. As manchas podem danificar toda a pele do corpo e o revestimento da boca e dos olhos. Nos casos mais graves, toda a pele é descolada e há necessidade de internação em unidade de terapia intensiva. Os rins e o fígado também podem ser afetados (Pires et al, 2017).

O diagnóstico da farmacodermia muitas vezes é difícil pela grande multiplicidade de aspectos clínicos e pela semelhança com outras doenças, sendo que as primeiras providências incluem a suspensão do medicamento em uso e a procura imediata de auxílio médico. Antialérgicos de uso comum não são imediatamente eficazes, sendo necessário determinado tempo para a melhora completa dos sintomas até a eliminação total do fármaco problema do organismo (Santos et al., 2015).

Tavares et al (2016), descreve que felizmente a maioria dos pacientes com farmacodermias tem uma recuperação após suspensão do fármaco e acompanhamento por profissionais da área da saúde por 12 dias, em média. Após 6 meses de tratamento paciente apresentou remissão completa do quadro.

\section{Metodologia}

O trabalho foi elaborado através de uma revisão sistemática de literatura, segundo Mattos (2015) sobre a farmacodermia. A pesquisa foi realizada utilizando a palavra-chave farmacodermia, nos portais Google Acadêmico e Portal Regional da BVS (Biblioteca Virtual em Saúde) que utilizam as bases de dados do Scientific Electronic Library Online (SCIELO), Literatura Latino-Americana e do Caribe em Ciências da Saúde (LILACS), Medical Literature Analysis and Retrieval System Online (MEDLINE) entre outras, resultando em 153 trabalhos científicos.

Os critérios de inclusão na pesquisa foram trabalhos publicados nos últimos cinco anos (2015-2020), no idioma português, disponíveis na íntegra e relacionados a saúde humana. Após a exclusão de artigos duplicados e que não atenderam aos critérios de inclusão, foram selecionados para elaboração dessa pesquisa 35 artigos, conforme Figura 1.

\section{Resultados e Discussão}

As publicações selecionadas para este estudo compreendem o período de 2015 a 2020, do s qua is 08 (18,69\%) artigos são do ano de 2015, 04 (9,03\%) artigos em 2016, 08 (27,90\%) artigos em 2017, 4 (11,62\%) artigos em 2018, seguido de 10 (25,58\%) artigos em 2019 e por fim 03 (6,78\%) artigos em 2020, conforme o Figura 2. 
Figura 1. Detalhamento dos artigos incluídos na revisão.

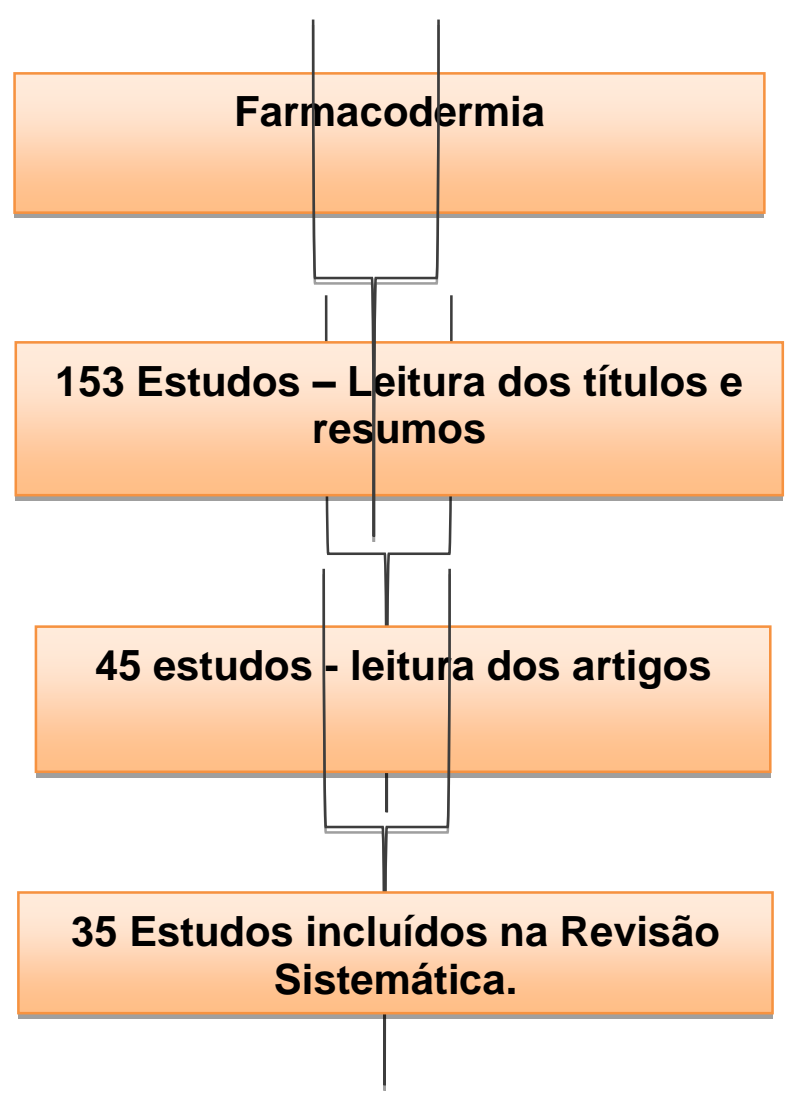

Fonte: Elaborada pelos autores (2020).

Figura 2. Número total de estudos incluídos na pesquisa por ano.

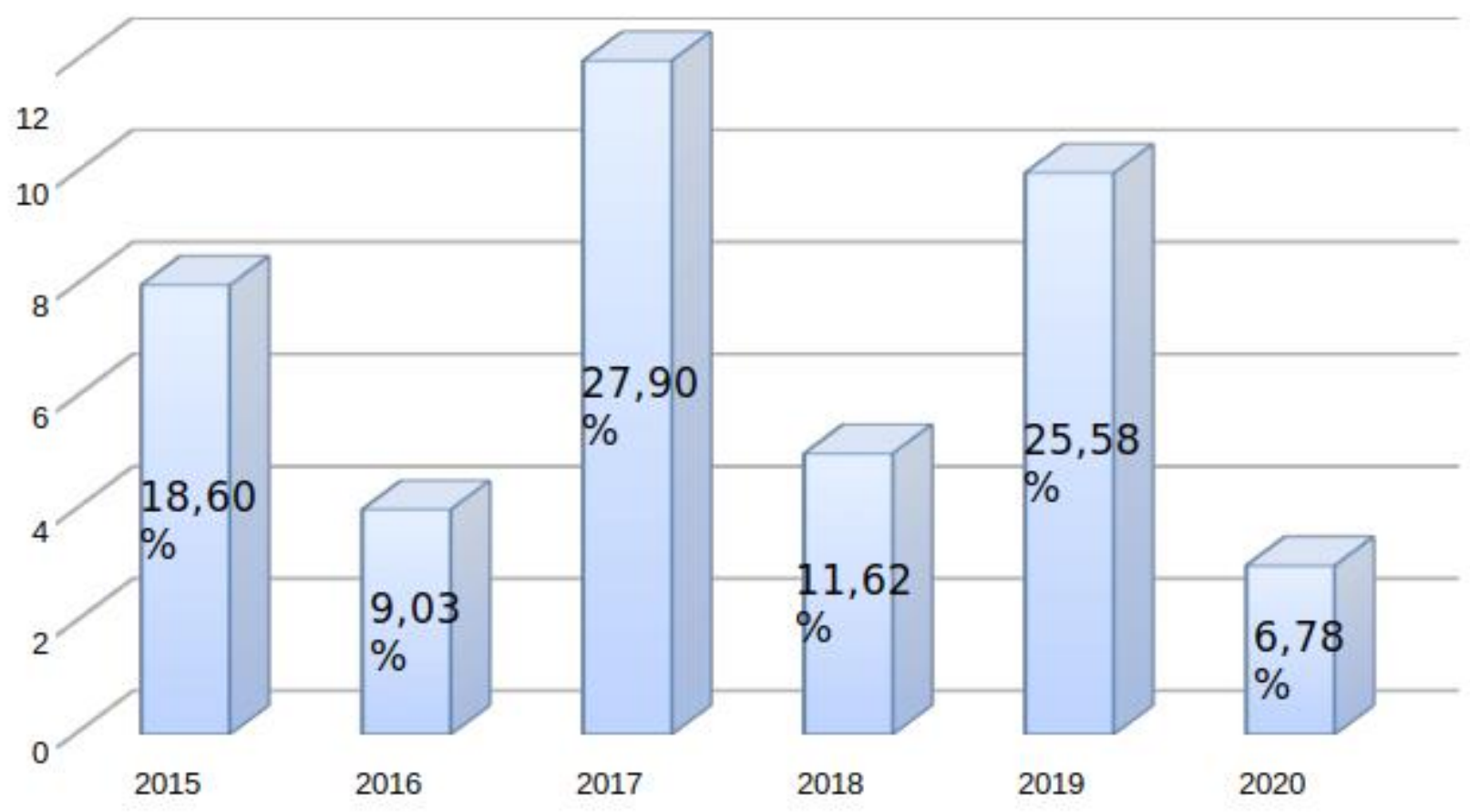

Fonte: Elaborada pelos autores (2020). 
Quanto aos tipos de publicações, Relato de caso esteve presente em todos os anos de publicações, totalizando 14 publicações (46,51\%), seguido de Pesquisa de campo com 12 artigos $(27,90 \%)$ das publicações estudadas ao logo de 2015 a 2020.

Os demais tipos de publicações encontradas foram 03 artigos (13,95\%) de Revisão de literatura, 04 artigos (6,97\%) de Revisão sistemática e 02 artigos $(4,65 \%)$ de Revisão de literatura narrativa, conforme demostrado na Figura 3.

Figura 3. Tipos de estudos analisados.

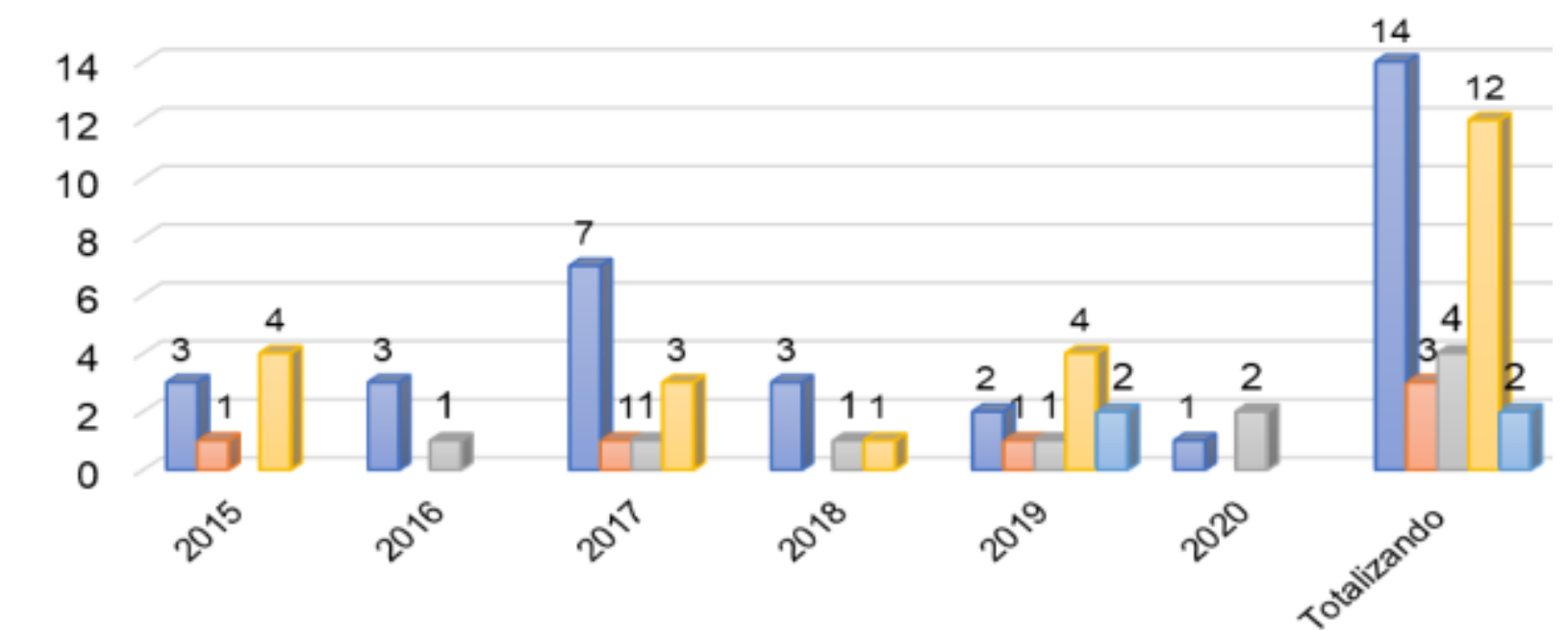

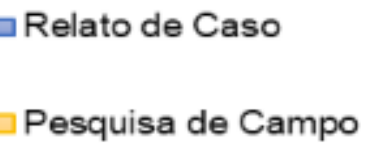

$\square$ Revisão Sistemática

$\square$ Revisão de Literatura Narrativa

Fonte: Elaborada pelos autores (2020).

Dos artigos publicados com a metodologia Relato de caso, verificou-se que a maioria dos trabalhos investigados foram casos de pacientes do sexo feminino, 12 casos $(63,15 \%)$ e 07 casos $(36,85 \%)$ de paciente do sexo masculino, conforme o Figura 4. A média de idade dos pacientes do sexo feminino foi de 46,9 e 48 anos do sexo masculino conforme Figura 4.

Figura 4. Prevalência de gênero nos trabalhos de estudo de caso.

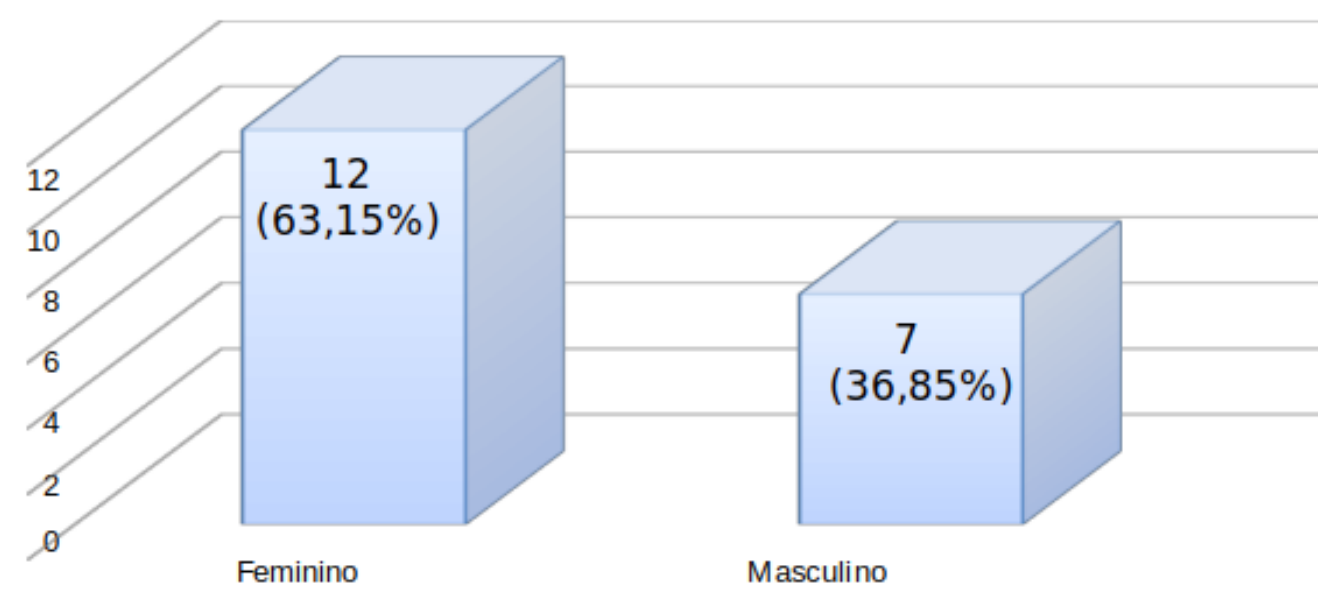

Fonte: Elaborada pelos autores (2020). 
Os principais fármacos responsáveis por farmacodermias nos últimos cinco anos e seus principais sinais e sintomas relacionados em estudos de caso foram descritos na Tabela 1.

Tabela 1. Principais Fármacos responsáveis por Farmacodermias e sintomas relacionados.

\begin{tabular}{|c|c|c|c|}
\hline Autor & Ano & Medicação utilizada & Principais Sinais e Sintomas \\
\hline Bispo Junior et al. & 2015 & Quimioterapia Antineoplásica Bleomicina. & $\begin{array}{l}\text { Hiperpigmentação, placas eritemato-violáceas, algumas descamativas, outras } \\
\text { com vesículas e bolhas na superfície, de configuração linear, disseminadas no } \\
\text { pavilhão auricular, membros e tronco. }\end{array}$ \\
\hline Meira Junior et al. & 2015 & Ácido Valproico, Lamotrigina, Fluimucil & $\begin{array}{l}\text { Febre e exantema máculo-papular inicialmente na face, que depois se } \\
\text { generalizou. }\end{array}$ \\
\hline Canabrava e Soares & 2016 & Paracetamol; Prometazina. & $\begin{array}{l}\text { Eritema difuso com áreas de exulceração e descamação em fronte e dorso } \\
\text { nasal Presença de liquenificação com algumas fissuras, além de eritema } \\
\text { difuso e áreas de exulceração no colo, na região cervical posterior e nos } \\
\text { membros superiores. }\end{array}$ \\
\hline Tavares et al. & 2016 & AINH & $\begin{array}{l}\text { Lesões eritemato-papulosas, disseminadas, intensamente pruriginosas, que } \\
\text { coalesceram formando placas nodulares. }\end{array}$ \\
\hline Quaresma et al. & 2016 & Dipirona & $\begin{array}{l}\text { Máculas hipercrômicas, castanho-a- cinzentadas, ovaladas, bem delimitadas e } \\
\text { simétricas nas regiões periorbitárias bilateralmente. }\end{array}$ \\
\hline Kohler et al. & 2017 & $\begin{array}{l}\text { Sulfametoxazol + Trimetoprima e } \\
\text { Corticoide }\end{array}$ & Diarreia, febre, dor abdominal e hepatoesplenomegalia. \\
\hline Nassar e Oliveira & 2017 & Naproxeno & Máculas, que evoluíram para pápulas e vesículas associando-se a prurido. \\
\hline Silva et al. & 2017 & Sulfametoxazol-trimetoprina & $\begin{array}{l}\text { Exantema pruriginoso difuso, exsudato mucopurulento em orofaringe e } \\
\text { pilares amigdalianos. Evolui com piora do exantema, deterioração } \\
\text { neurológica com confusão mental, redução no nível de consciência, } \\
\text { desorientação, marcha táxica, movimentos coreiformes em membros } \\
\text { superiores, leve edema em face e região cervical. }\end{array}$ \\
\hline Ferreira et al. & 2017 & Carbamazepina & $\begin{array}{l}\text { Febre, rash eritemato-maculopapuloso difuso, com edema centro-facial e } \\
\text { linfonodos }>2 \mathrm{~cm} \text {, móveis, em região cervical. }\end{array}$ \\
\hline Borges et al. & 2019 & Hidroxicloroquina & $\begin{array}{l}\text { Febre, de dermatite eritemato-bolhosa nas axilas que rapidamente evoluiu } \\
\text { para lesões eritemato-bolhosa-descamativas em todo o corpo, incluindo face, } \\
\text { poupando áreas de mucosa. }\end{array}$ \\
\hline $\begin{array}{l}\text { Caravanti, Vendrame e } \\
\text { Aguiar. }\end{array}$ & 2019 & Vitamina D3 & Lesão única, pápulo-eritematosa em região malar esquerda. \\
\hline $\begin{array}{c}\text { Franco, Fonseca e } \\
\text { Hassan }\end{array}$ & 2019 & Carbamazepina & $\begin{array}{l}\text { Presença de edema, liquenificação e queratinização com presença de escamas } \\
\text { foliáceas, predominante. }\end{array}$ \\
\hline Lonchiati et al. & 2020 & $\begin{array}{l}\text { Levofloxacino, ciprofloxacino, } \\
\text { clindamicina, cefalexina, cilostazol, } \\
\text { clopidogrel, hidroclorotiazida, metildopa, } \\
\text { sinvastatina e ácido acetilsalicílico. }\end{array}$ & $\begin{array}{l}\text { Presença de bolhas tensas íntegras e rotas, crostas e máculas hipocrômicas em } \\
\text { pernas, antebraços e mãos (áreas fotoexpostas). }\end{array}$ \\
\hline
\end{tabular}

Fonte: Elaborada pelos autores (2020).

Os trabalhos de pesquisa de campo obtiveram uma variação quanto ao número de pacientes estudados, situando-se entre 10 e 300 pacientes analisados, o gênero predominante foi do sexo masculino e a variação de idade foi de 18 a 95 anos. 
Verificamos que as classes farmacológicas de maior incidência em casos de farmacodermias foram os antiinflamatórios, quimioterápicos, anticonvulsivantes, ansiolíticos, anti-hipertensivos e antibióticos. Notamos também, que em alguns casos, ocorreu suspeita de uso concomitante de mais de um medicamento, indicando polifarmácia, que evidencia a dificuldade de identificar o causador das farmacodermias nesses casos.

Marques et al. (2016) através de revisão de literatura, apontaram que as reações de hipersensibilidade imediata a penicilina é causada por mudanças na estrutura química do fármaco que não passam despercebidas pelo sistema imune. Essas reações necessitam de tratamento cauteloso, pois a reação anafilática pode levar a óbito rapidamente sem o tratamento imediato e adequado.

Andrade et al. (2019), ao realizar revisão bibliográfica sobre Síndrome de Steven- Johnson, com destaque na Terapia Intensiva, notaram que essa síndrome é uma doença de hipersensibilidade tardia e pouco frequente. Sua etiologia não é bem definida, podendo se relacionar com fármacos em $50 \%$ das vezes, sendo a classe farmacológica anticonvulsivantes a mais relatada. Já os fármacos, de diferentes classes farmacológicas, mais citados são alopurinol, hidantoína, piroxicam, carbamazepina e sulfas. Outros pontos relevantes a serem citados, o diagnóstico deve ser clínico (ou sindrômico) e a biópsia é indicada em casos atípicos, podendo ser potencialmente fatal quando não diagnosticada precocemente.

Visentainer e Visentainer (2020), ao averiguar as manifestações cutâneas na COVID-19, notaram através de dados existente na literatura que o SARS-CoV-2 pode afetar vários órgãos e, possivelmente, a pele. Contudo, existem poucos trabalhos publicados sobre manifestações cutâneas na COVID-19, sendo esses, em sua maioria, relatos de casos. As manifestações cutâneas descritas são rash, apresentando vermelhidão inespecífica na região do tronco, urticária e lesões que foram confundidas com um quadro de dengue.

Através de revisão sistemática, Pereira et al. (2019), ao avaliar as evidências científicas relacionadas à identificação, prevenção e tratamento da síndrome mão-pé induzida por agentes quimioterápicos, identificaram os principais sinais e sintomas que possibilitam o reconhecimento da síndrome. Notaram que existem evidências consistentes, porém não contemplam todos os fármacos indutores da síndrome e não exploram outras manifestações relacionadas às onicólises e onicomicoses. O estudo apresentou resultados que poderão auxiliar os prescritores na identificação da síndrome mão-pé, além de alternativas para prevenção e tratamento. Contudo, vale destacar a necessidade de pesquisas futuras para elucidar a etiologia e protocolos de tratamento.

Roviello et al. (2019), ao elaborarem revisão de literatura narrativa, com o intuito de identificar as manifestações clínicas da necrólise epidérmica tóxica e síndrome de Stevens-Johnson, concluíram que a patogênese da necrólise epidérmica tóxica e Síndrome de Stevens-Johnson se dá pela hipersensibilidade tardia a fármacos. A principal manifestação clínica é o aparecimento de eritema cutâneo e ambas as patologias são farmacodermias graves, com baixas incidências, mas elevada mortalidade, assim o reconhecimento precoce das doenças e a retirada do fármaco causador são essenciais para conduzir o tratamento, diminuindo por sua vez a taxa de mortalidade.

Souza e Ferreira Neto (2019) com o objetivo de nortear a identificação e manejo de graves reações adversas cutâneas a medicamentos, concluíram, através de revisão de literatura narrativa, que o conhecimento das reações adversas a medicamentos permite a correta investigação da reação adversa e manejo adequado. A existência de documentos institucionais com as informações necessárias para execução da Farmacovigilância, fornecendo o embasamento científico necessário para tomada de decisões e manejo de quadros de incidentes relacionados a medicamentos irão garantir a segurança do paciente. 


\section{Considerações Finais}

Verificamos que as classes farmacológicas de maior incidência em casos de farmacodermias foram os antiinflamatórios, quimioterápicos, anticonvulsivantes, ansiolíticos, anti-hipertensivos e antibióticos, contudo toda medicação pode causar efeitos adversos do tipo farmacodermias. Essas classes estão entre as mais prescritas em todo mundo, portanto, o contato da equipe multiprofissional e a farmácia clínica são essenciais para a rápida identificação de possíveis farmacodermias.

Os principais sintomas relacionados às farmacodermias foram: febre; edema; lesões eritematosas; placas eritematovioláceas; bolhas na superfície da pele; hiperpigmentação; eritema difuso com áreas de exulceração, máculas hipercrômicas; rash eritemato-maculopapuloso difuso; lesões cutâneas eritematosas, máculo-papulares, difusas e pruriginosas; eritema multiforme; liquenificação e queratinização com presença de escamas foliáceas, predominante na área afetada e ainda presença de bolhas tensas íntegras e rotas, crostas e máculas hipocrômicas e fotossensibilidade.

As farmacodermias possuem baixa incidência, porém elevada mortalidade, por isso o reconhecimento precoce e a retirada do fármaco causador são essenciais na condução do tratamento. Portanto, é fundamental o contato do paciente com equipe multiprofissional e a farmácia clínica para a rápida identificação de possíveis farmacodermias.

Mais estudos abordando o tema da Farmacodermia devem ser realizados para aprimorar o conhecimento na área de farmacêuticos e demais profissionais de saúde.

\section{Agradecimentos}

Agradecemos a Marilza Aparecida Machado Borges pelo auxílio a correção gramatical.

\section{Referências}

Andrade, B. C. B., Cunha, D. F., Silva, M. A. P., \& Soares, T. A. G. (2019). Farmacodermia em terapia intensiva: uma revisão bibliográfica de Síndrome de Steven Johnson. Rolim de Moura: Revista Saberes da Faculdade São Paulo - FSP - 9 (1).

Bispo Junior, et al. (2015). Dermatite Flagelada Induzida por Bleomicina: Relato de Caso. Cogitare Enfermagem, 20 (2). https:// revistas.ufpr.br/cogitare/article/view/39428>.

Borges, R., Farias, L., Andrade, R., Costa, E., Sousa, A., \& Aires, M. (2019). Necrólise epidérmica tóxica secundária ao uso de hidroxicloroquina em paciente com Chikungunya: relato de caso. Revista da Faculdade de Ciências Médicas de Sorocaba, 21 (1), $42-44$.

Canabrava, P. B. E. \& Soares, S. K. P. (2016). Farmacodermia e Dermatite de Contato Alérgica Crônica, Revista de Medicina e Saúde de Brasília, 5 (3).

Caravanti, N. C., Vendrame, C. B., \& Aguiar, B. S. (2019). Hiperplasia linfoide cutânea de padrão reacional (pseudolinfoma): Relato de caso, Revista Corpus Hippocraticum, 1 (1).

Capeli, R. L. A. (2015) Hepatite C crônica e manifestações cutâneas: experiência no acompanhamento conjunto (demartologia / hepatologia) dos pacientes em tratamento para hepatite C. Dissertação (Mestrado em Medicina) - Universidade Federal do Espírito Santo, Centro de Ciências da Saúde, Vitória.

Dantas, J. M. S. (2015). Estudo clínico-epidemiológico das dermatoses em pacientes HIV-positivo atendidos em um centro de referência no Piauí. $152 \mathrm{f}$. Dissertação (Mestrado em Medicina Tropical) - Fundação Oswaldo Cruz, Instituto Oswaldo Cruz, Rio de janeiro, RJ, 2015.

Figueiredo, I. R., Azevedo, A. R. S., Carvalho, L. A. D., Lawall, A. R. N., Silva, D. O. F., Castro, U. R. (2017). Farmacodermia induzida por Bupropiona em paciente com transtorno depressivo maior. Brasília: Revista de Medicina e Saúde de Brasília, 6 (3), $334-340$.

Ferreira, M. I. P. L., Silva, E. C. F., Pôrto, L. C., Alves, M. F. G. S., Arraes, A. C., Castro, A. M. (2017). Dress: Relato de caso com estudo genético. Arq Asma Alerg Imunol., 1 (4), 417-421.

Franco, M. S. B., Fonseca, B. S., Hassan, S. E. L. (2019). Reações cutâneas associadas ao uso da carbamazepina, Revista Corpus Hippocraticum, 1 (1).

Gomes, V. R. (2017) Eventos adversos a medicamentos em idosos de unidades de terapia intensiva. São Paulo, Dissertação de mestrado, Gomes, Vanessa Rossato, 134 p. https://www.teses.usp.br/teses/disponiveis/7/7139/tde-27042018-143325/publico/VanessaRossato GomesCorrigida.pdf

Innocencio, G. T. de C., Martins, M. L., Orioli Da Silva, C. de P., Valente, T. L., Coelho Innocencio, M. F., \& Almeida De Souza, M. C. (2017). Síndrome do Eritema multiforme causado por Farmacodermia. Revista De Saúde, 8 (1), 45-47.

Kikuchi, H. M. T., Suguimatsu, L. C. F., \& Santos, R. V. (2018). Reações de Hipersensibilidade a Drogas: Estudo Epidemiológico em um Hospital de Referência de Curitiba. Rev. Méd. Paraná, Curitiba, 76 (2), 56-72. 
Kohler, L. I. A., Azevedo, J., Caetano, L. M., Machado, L. N., Souza, L. A., Lima, M. A., \& Souza, L. J. (2017). Acometimento visceral e ocular simultâneo em infecção por toxocara canis acompanhados de farmacodermia. Rev. Soc. Bras. Clín. Méd., 15 (2), $112-115$.

Lima, M. T. A., Mazzoni, J. R. B., Gonçalves, R. DO A., Vicente Pereira, L. L., \& De Godoy, M. F. (2019). Estudo da utilização de esteroides anabólicos androgênicos e suplementos alimentares por universitários em São José do Rio Preto-SP. Revista Brasileira De Nutrição Esportiva, 13 (79), $333-339$.

Lonchiati, D. et al. (2020). Penfigóide bolhoso induzido por fármaco: um relato de caso. Colloquium vitae, 12 , 6-11.

Marques, A. C., Silveira, T. C., \& Borges, R. C. (2016). Hipersensibilidade imediata associada à penicilina. In: Simpósio de TCC e Seminário de IC, 2016.

Mattos, P. C. (2015). Tipos de Revisão de Literatura. Faculdade de Ciências Agronômicas UNESP, Campus Botucatu.

Meira Júnior, J. D., Souza, J. T., Toniollo, C. F., \& Stolf, H. O. (2015). Necrólise epidérmica tóxica/síndrome de Stevens-Johnson: emergência em dermatologia pediátrica. Revista Diagnóstico e Tratamento, 20 (1), 8-13.

Nassar, A. A. \& Oliveira, A. C. (2017). Farmacodermia por Naproxeno: Um relato de caso. Revista de Saúde, 8(1), $112-113$.

Naspoline, A. P. et al. (2015) Necrólise epidérmica tóxica: relato de caso com indicação de imunoglobulina endovenosa, Hospital de Clínicas de Porto Alegre, Semana Científica (35), 2015. https://lume.ufrgs.br/handle/10183/140911

Nepomuceno, B. B. et al. (2018). Necrólise Epidérmica Tóxica desencadeada por Amoxicilina: Manejo da dor, 4 (2), Anais do VI Simpósio Multiprofissional em Saúde, 2018.

Organização Mundial da Saúde (OMS, 2015). Segurança dos medicamentos: um guia para detectar e notificar reações adversas a medicamentos. Por que os profissionais de saúde precisam entrar em. A. (2019). Identificação, Prevenção e Tratamento da Síndrome Mão-Pé Induzida por Quimioterapia: Revisão Sistemática, Revista Brasileira de Cancerologia, 65 (4), e-05363.

Pires, A. B., Madeira, A. C. A., D’araújo, K. M., Souza Grossi, L. D., Valadão, A. F., Motta, P. G. (2017). Reações adversas na cavidade oral em decorrência do uso de medicamentos. Rev. Saluv., 36 (1), 157-185.

Quaresma, M. V., de Abreu, L., Oliveira, L. B., Poggi, B., de Rezende, T. V., \& Ravo, B. S. F. (2016). Erupção medicamentosa fixa na face associada a dipirona: correlação dos achados clínicos, histopatológicos e dermatoscópicos. Rio de Janeiro: Surgical \& Cosmetic Dermatology, 8 (4), 352-356.

Rodrigues, M. T. T., Cabral, G. C. C., Ferro, I. A. M. ação. OMS, 2-4.

Pereira, P. P., Pedroso, R. R., \& Ribeiro, M. A. (2019). Identificação, Prevenção e Tratamento da Síndrome Mão-Pé Induzida por Quimioterapia: Revisão Sistemática, Revista Brasileira de Cancerologia, 65 (4), e-05363.

Pires, A. B., Madeira, A. C. A., D’araújo, K. M., Souza Grossi, L. D., Valadão, A. F., Motta, P. G. (2017). Reações adversas na cavidade oral em decorrência do uso de medicamentos. Rev. Saluv., $36(1), 157-185$.

Quaresma, M. V., de Abreu, L., Oliveira, L. B., Poggi, B., de Rezende, T. V., \& Ravo, B. S. F. (2016). Erupção medicamentosa fixa na face associada a dipirona: correlação dos achados clínicos, histopatológicos e dermatoscópicos. Rio de Janeiro: Surgical \& Cosmetic Dermatology, 8 (4), $352-356$.

Rodrigues, M. T. T., Cabral, G. C. C., Ferro, I. A. M., Guimarães, M. G., \& Cabral, H. L. T. B. (2018). Eritema Multiforme Induzido por Fenobarbital: Relato de Caso, Interdisciplinary Scientific Journal, 5 (6).

Rossi, G. (2017). Aspectos Clínicos e Dermatoscópicos das Farmacodermias - Dissertação (Mestrado) - Universidade Federal do Rio Grande do Sul, Faculdade de Medicina, Programa de Pós-Graduação em Medicina: Ciências Médicas, Porto Alegre.

Roviello, C. F., Rodrigues, F. S. M., Gonçalves, J. A. B., \& Ferraz, R. R. N. (2019). Manifestações e tratamento da necrólise epidérmica tóxica e da síndrome de Stevens Johnson, Journal Health NPEPS, 4 (1), 319-329.

Santos, F. P., Quitério, L. M., Pinto, V. B., \& Gomes, L. B. (2015). Farmacodermia: Identificação dos tipos, medicamentos envolvidos e classes farmacológicas que acometem pacientes internados na clínica dermatológica. São Paulo: Revista Brasileira de Farmácia Hospitalar e Serviços de Saúde, 6 (2), 12-17.

Silva, J. M., Tiago, V. P., Juliano, C. B., Cunali, V. C. A., \& Bonatti, R. C. F. (2017). Necrólise epidérmica tóxica induzida por sulfametoxazol-trimetoprina associado à lesão cerebral. Resid. Pediatr., 7 (1), 17-20.

Sociedade Brasileira de Dermatologia (2017). Sua Saúde. Rio de Janeiro: 2017. http://www.sbd.org.br/dermatologia/pele/doencas-e-problemas/ farmacodermia/27

Souza, I. V. \& Ferreira Neto, C. J. B. (2019). Informações Essenciais para Identificação, Avaliação e Manejo de Farmacodermias, Ensaios e Ciênc., 23 (3), 219-229.

Tavares, L. P. S., Potenciano, O., Pugliesi, Y., Junior, J. A. L. F., Freitas, D. M., Araujo, A. C. V., TOSE, T. B., Potenciano, N. L. B. C., Marques, A. M., Paixão, I. S., \& Godinho, G. A. (2016). Reação cutânea desencadeada por droga: Relato de Caso. Palmas: Revista de Patologia do Tocantins, 3 (4), $130-134$.

Valviesse, V. R. G. A., Pinho, L. I., Freitas, R. F., Varon, A. G., \& Lemos, A. S. (2017). Hipersensibilidade a lamivudina em paciente infectada pelo HIV: relato de caso. Medicina Ribeirão Preto. Online.

Visentainer, L. \& Visentainer, J. (2020). Manifestações cutâneas na covid-19, Departamento de Ciências Básicas da Saúde da Universidade Estadual de Maringá (UEM), 2020. 